\title{
X-ray emission of interacting wind binaries in Cyg OB2
}

\section{Abstract}

Cyg OB2 \#5, \#8A, and \#9 are binary or multiple massive stars in the Cyg OB2 association displaying several peculiarities, such as bright X-ray emission and non-thermal radio emission. Our X-ray monitoring of these stars reveals the details of their behaviours at high energies, which can be directly linked to wind-wind collisions (WWCs). In addition, the X-ray emission of Cyg OB2 \#12, an evolved massive star, shows a long-term decrease, which could hint at the presence of a companion (with associated colliding winds) or indicate the return to quiescence of the system following a recent eruption.

References: Nazé et al, A\&A, 546, A37 (2012) and Cazorla et al, A\&4, submitted.

\section{Data}

Our dataset comprises 7 XMM and 6 Switt observations of Cyg OB2, which were respectively processed using SAS v12.0.0 and HEASOFT v6.13. Archival Suzaku and R.OSAT data were also used. Extracted spectra were fitted using absorbed opticallythin thermal plasma models. The fitting was performed in two steps: first, a fully-free fitting of the XMM data (those with highest $S / N$ ), followed by a second fitting after fixing all apparently constant parameters.

\section{Crg OB2 \#5}

Cyg OB2 \#5 was recently found to be a quadruple system (Kennedy et al. 2010) consisting of an eclipsing binary with $6.6 \mathrm{~d}$ period, a tertiary star in a 6.7 yrs orbit around it, and a fourth object at $0.8^{\prime \prime}$. The X-ray emission can be fitted with two thermal components, with constant temperatures and ratio between the normalization factors. However, both flux and absorption vary. The flux has a complex behaviour, incompatible with changes over a $6.6 \mathrm{~d}$ period; the Xray emission rather appears to be modulated by the 6.7vis period: it is thus associated with the wind-wind collision between the binary and the third star, as is the radio emission. This is supported by the variation of the absorption since the latter appears stronger when the binary is in front. Since the collision should be adiabatic, its theoretical behaviour is known and our study therefore allowed us to test (and discard) some orbital solutions proposed for this triple system.

Reflex motion induced by the third star was detected for eclipse times and radial

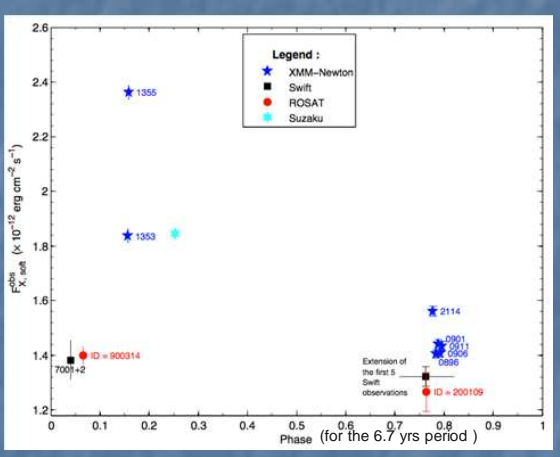
velocity curves of the binary, but additional data must be gathered before a secure solution is achieved.

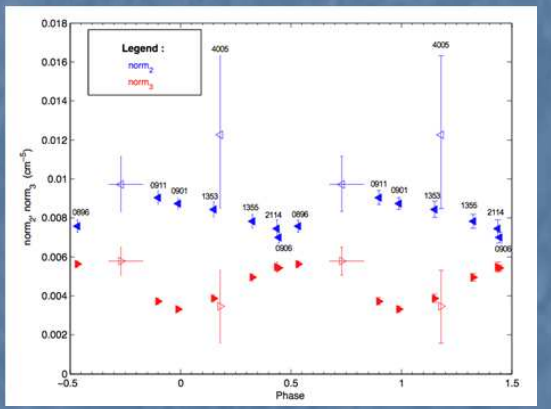

\section{CYa OB2 \#9}

Despite the fact that it was one of the first non-thermal radio emitter discovered amongst O-stars, Cyg OB2 $\# 9$ was only found to be a binary in 2008: the period amounts to $860 \mathrm{~d}$ and the eccentricity to 0.7 . Since then, the first observable periastron passage occurred in 2011, and a large multiwavelength campaign was organized to follow the system behaviour.

In X-rays, Oyg 0B2 $\$ 9$ dosely follows the expectations for an adiabatic collision, i.e. LX $\propto 1 / D$ : there is no crash of the collision zone on one of the two stars, and the collision remains mostly adiabatic in nature even when stars are closest, which renders the system exceptional. Moreover, the temperature of the hottest plasma decreased by $25 \%$ at periastron. This can be explained if radilitive braking is taken into account:
Cyg OB2 \#8A is a binary with a period of $21.9 \mathrm{~d}$ and a radiative wind-wind collision (De Becker et al. 2006 , Blomme et al. 2010). Our work enlarges the phase coverage of the orbit. We confirm the previous results reported for the system: slight temperature increase at apastron (due to larger wind speeds at that phase), maximum absorption at periastron (when the star with strongest wind is in front), and maximum flux at an intermediate phase. The strength of the cooler component varies with density (hence increases at periastron) whereas that of the hotter component varies with shock strength (hence increase at apastron). It should be noted that the flux variations are asymmetric, displaying the hysteresis predicted from hydro modes (Pittard \& Parkin 2010).

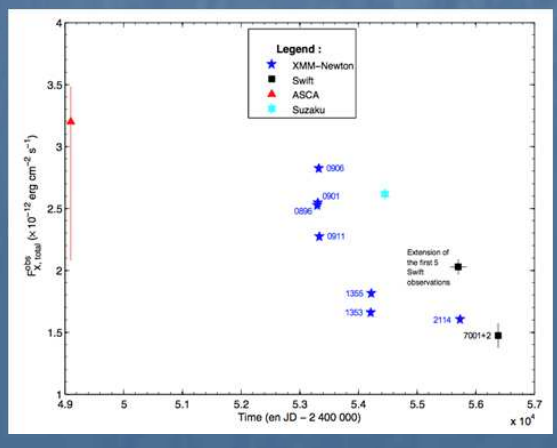

\section{Gra OB2 \#12}

Cyg OB2 \#12 is one of the optically brightest stars in the Galaxy and an LBV candidate, though it lacks some of the typical LBV characteristics (Clark et al. 2012). Best-fit was achieved with two thermal components with constant temperatures and ratio between normalization factors. The overall flux has been decreasing during the last decade, which could be explained considering either the aftermath of an LBV eruption or the decline of the emission of an adiabatic wind-wind collision in a longperiod binary (after periastron). Archival ROSAT and ASCA data indicate a binghtening of the system in the 1990s: this is consistent with the second scenario if the binary period is longer than $24 \mathrm{yr}$. Additional monitoring is now required to better characterize this system. 